\title{
Ni-Catalyzed Alkyne Cycloaddition to Give Boronic Esters
}

Key words

benzannulation

aromatic boronic esters

nickel<smiles>[R7]C1=CC(=O)C1</smiles><smiles>[R]C#CB1OC2(C)CC3CC1(C3)O2</smiles>

$\left[\mathrm{Ni}(\mathrm{cod})_{2}\right](10 \mathrm{~mol} \%)$

$\mathrm{Et}_{2} \mathrm{O}, 16 \mathrm{~h}, 0^{\circ} \mathrm{C}$ to r.t.

$\mathrm{R}^{1}=n-\mathrm{Bu}, \mathrm{Ph}$, cyclohex-1-enyl

$\mathrm{R}^{2}=\mathrm{Me}, \mathrm{H}$

$\mathrm{R}^{3}=\mathrm{TMS}, n-\mathrm{Bu}, \mathrm{Ph}$, cyclohex-1-enyl, $\mathrm{CH}_{2} \mathrm{OBn}$<smiles>[R7]c1c(Br)cc(O)c([10BH2])c1Br</smiles>

isomer $\mathbf{A}$<smiles>Oc1cc(P)c(P)c(Br)c1Br</smiles>

isomer $\mathbf{B}$

up to $92 \%$ yield

Selected examples:<smiles>CCCCc1cc(O)c(S(C)(=O)=O)c(Br)c1CCCC</smiles>

$81 \%$ yield ratio $\mathbf{A} / \mathbf{B}>98: 2$<smiles>CCCCc1c(O)cc(-c2ccccc2)c(C)c1Cc1ccccc1</smiles>

$68 \%$ yield ratio $\mathbf{A} / \mathbf{B}>98.2$<smiles>Cc1c(-c2ccccc2)cc(O)c(Br)c1C1=CCCCC1</smiles>

$57 \%$ yield ratio $\mathbf{A} / \mathbf{B}=20: 80$<smiles>Oc1cc(-c2ccccc2)cc(-c2ccccc2)c1Br</smiles>

$67 \%$ yield ratio $\mathbf{A} / \mathbf{B}=44: 56$<smiles>Oc1cc(C2=CCCCC2)cc(C2=CCCCC2)c1Cc1ccccc1</smiles>

$61 \%$ yield ratio $\mathbf{A} / \mathbf{B}=31: 69$<smiles>CCCCc1c(O)cc(C2=CCCCC2)cc1Br</smiles>

$92 \%$ yield ratio $\mathbf{A} / \mathbf{B}>98.2$<smiles>CCCCc1cc(O)c(COCc2ccccc2)c(Br)c1CCCC</smiles>

$48 \%$ yield ratio $\mathbf{A} / \mathbf{B}=93: 7$
Significance: A new nickel-catalyzed benzannulation of cyclobutenones with alkynylboronates to yield aromatic boronic esters has been disclosed. Furthermore, a one-pot cycloaddition-coupling protocol has been developed, whereby the nickel catalyst performs firstly the benzannulation and afterwards the cross-coupling.
Comment: Alkynylboronates bearing $\mathrm{sp}^{3}$-based substituents show high selectivity for isomer $\mathbf{A}$, whereas those bearing $\mathrm{sp}^{2}$-based substituents mainly favor isomer $\mathbf{B}$ 